\title{
MICROBIOLOGICAL FLORA AND THEIR ANTIBIOTIC SUSCEPTIBILITY- A STUDY UNDERTAKEN WITH ENDOTRACHEAL TUBE TIPS AND ENDOTRACHEAL ASPIRATES IN A TERTIARY CARE HOSPITAL IN KOLKATA
}

\author{
Rina Das ${ }^{1}$ Indrani Bhattacharyya ${ }^{2}$
}

${ }^{1}$ Assistant Professor, Department of Microbiology, Calcutta National Medical College, Kolkata, West Bengal, India.

${ }^{2}$ Associate Professor, Department of Microbiology, Calcutta National Medical College, Kolkata, West Bengal, India.

\begin{tabular}{l}
\hline ABSTRACT \\
BACKGROUND \\
Endotracheal (ET) tubes are life saving devices on one hand and life takers on the other. This study aims to find out how morbidity \\
in the form of nosocomial pneumonia can be predicted to save patients and to minimise expenditure.
\end{tabular}

\section{MATERIALS AND METHODS}

This was a descriptive study. ET tube tips, tracheal aspirates and laryngeal aspirates were collected and tested from patients intubated for a minimum of 48 hours. 31 such samples were processed by culture in MacConkey Agar and Blood Agar media. Growth was observed.

\section{RESULTS}

The results showed the growth of Klebsiella Pneumoniae (54.1\%) and Acinetobacter baumannii (33.3\%). These findings are similar to several other studies in different parts of India e.g. by Chandra Mouli et al, Dipti Chandra et al 2017 etc. and earlier by Elek and Conen in 1957. Antibiogram by Kirby Bauer method showed that all the organisms were multidrug resistant.1,2,3,4

\section{CONCLUSION}

This study reinforces the finding that ET tubes are a very important cause of pneumonia. Gram negative organisms in this study are the causative agents. Most of the organisms were sensitive to Colistin (except Proteus mirabilis, which is known to be intrinsically resistant). Moreover, the medical fraternity must be aware that on one hand the infections need immediate and correct intervention and on the other hand the resistance pattern shows that the choice of drugs is extremely limited and only the most toxic of them remain useful. Thus, the management of such cases has to be very carefully planned and balanced taking into consideration the immune status, age, co-morbidities and economic status of each patient.

\section{KEY WORDS}

Endotracheal Tube, Nosocomial Infection, Multidrug Resistant, Co-Morbidity, Virulence Factors, NICU and PICU.

HOW TO CITE THIS ARTICLE: Das R, Bhattacharyya I. Microbiological flora and their antibiotic susceptibility- a study undertaken with endotracheal tube tips and endotracheal aspirates in a tertiary care hospital in Kolkata. J. Evolution Med. Dent. Sci. 2018;7(25):2931-2933, DOI: 10.14260/jemds/2018/660

\section{BACKGROUND}

Personnel related to the medical profession are well aware that endotracheal tubes are mandatory in any instance requiring assisted respiration, which may be iatrogenic (as in general anaesthesia, collection of aspirate, biopsy etc.) or in diseases. On one hand intubation may be a life saver, i.e. an absolute necessity when the patient cannot breathe and on the other hand it is (more often than not) a major cause of nosocomial infection. The patients are ultimately saved at the cost of skilled professional help, a magnum expenditure and a huge toll on their already old and feeble bodies.

The cycle is usually the same severely ill patient followed by intubation, followed by nosocomial infection with MDR organism septicaemia. The fate is either, patients succumb (majority) or lucky ones respond and get well.5,6,7

'Financial or Other Competing Interest': None.

Submission 26-04-2018, Peer Review 02-06-2018,

Acceptance 08-06-2018, Published 18-06-2018.

Corresponding Author:

Dr. Indrani Bhattacharyya,

Aikataan, Flat No. B2, 37J/1D,

Raja Manindra Road,

Kolkata-700037,

West Bengal, India.

E-mail: indranichaudhuri@yahoo.com

DOI: $10.14260 /$ jemds $/ 2018 / 660$
The huge increase in hospital stay followed by increase in expenditure and many a time a fatal outcome at the end of it all lead us to undertake this study.

\section{MATERIALS AND METHODS}

A descriptive study was performed over six months from January 2017 to June 2017.

\section{Samples}

ET tubes- tips and aspirates. Samples were only those whose ET tubes were present in situ for 48 hours or more. Thirtyone such samples were obtained. Each sample was cultured aerobically in Blood agar and MacConkey agar. The plates were examined after 48 hours. Twenty-four samples were culture positive. Examination of colony characters, Gram stain and motility tests were done from the isolates. Biochemical reactions routinely performed in the laboratory including oxidation fermentation reaction in Hugh-Leifson media, catalase test, oxidase test, indole reaction, glucose, lactose mannitol and sucrose fermentation, citrate utilisation test, growth in triple sugar iron agar media, lysine, ornithine decarboxylase tests, arginine dihydrolase test, phenylpyruvic acid production tests were done. All isolates were Gram negative bacilli. 
RESULTS

The results were tabulated as follows:

\begin{tabular}{|c|c|c|}
\hline Serial No. & Sample Type & Number \\
\hline 1 & ET tube-tip & 28 \\
\hline 2 & Tracheal aspirate & 2 \\
\hline 3 & Laryngeal suction fluid & 1 \\
\hline Total & \multicolumn{3}{|c|}{} & \\
\hline Table 1. Details of Number of Samples Processed \\
\hline
\end{tabular}

\begin{tabular}{|c|c|c|c|}
\hline Sl. No. & Name of Bacteria & No. of Isolates & $\%$ \\
\hline 1 & Klebsiella pneumoniae & $13 / 24$ & $54.17 \%$ \\
\hline 2 & Acinetobacter baumannii & $8 / 24$ & $33.33 \%$ \\
\hline 3 & Proteus mirabilis & $1 / 24$ & $4.17 \%$ \\
\hline 4 & Citrobacter freundii & $1 / 24$ & $4.17 \%$ \\
\hline 5 & Pseudomonas aeruginosa & $1 / 24$ & $4.17 \%$ \\
\hline \multicolumn{3}{|c|}{ Table 3. Species of Bacteria Isolated } \\
\hline
\end{tabular}

\begin{tabular}{|c|c|c|c|}
\hline Sl. No. & Sample Type & Total No. & Culture Positive \\
\hline 1 & ET tube-tip & 28 & 22 \\
\hline 2 & Tracheal aspirate & 2 & 1 \\
\hline 3 & Laryngeal suction fluid & 1 & 1 \\
\hline Total & & $\mathbf{3 1}$ & $\mathbf{2 4}$ \\
\hline \multicolumn{3}{|c|}{ Table 2. Break-Up of Culture Positive Samples } \\
\hline
\end{tabular}

\section{Antibiotic}

\begin{tabular}{|c|c|c|c|c|c|c|c|c|c|c|}
\hline Organism & Mero & Pip-Tazo & AmC & Ctx & Levo/Cip & Do & Ak/Gen & C & PB & Cl \\
\hline Klebsiella pneumoniae & 2 & None & None & None & 3 & 2 & 3 & 3 & 13 & 13 \\
\hline Acinetobacter baumannii & 1 & None & None & None & 2 & 1 & 1 & 2 & - & 8 \\
\hline Citrobacter freundii & 1 & - & - & - & - & - & - & - & - & 1 \\
\hline Proteus mirabilis & 0 & None & None & None & None & 1 & None & None & None & None \\
\hline Pseudomonas aeruginosa & 1 & None & None & None & None & 1 & None & None & 4 & 1 \\
\hline \multicolumn{3}{|c|}{ Table 4. Sensitivity pattern of isolates } \\
\hline
\end{tabular}

Key- Mero= Meropenem, Pip-Tazo $=$ piperacillin-tazobactam, AmC $=$ amoxicillin-clavulanate, Ctx $=$ ceftriaxone, Levo $/ \mathrm{Cip}=$ levofloxacin/ ciprofloxacin, $\mathrm{Do}=$ doxycycline, $\mathrm{Ak} / \mathrm{Gen}=$ amikacin/ gentamicin, $\mathrm{C}=$ Chloramphenicol, $\mathrm{PB}=$ polymyxin $\mathrm{B}, \mathrm{CL}=\mathrm{Colistin}$.

\begin{tabular}{|c|c|c|c|}
\hline Ward & Klebsiella & Acinetobacter & Others \\
\hline PICU and NICU & 10 & 5 & 3 \\
\hline ICU & 1 & 0 & 0 \\
\hline \multicolumn{3}{|r|}{ Table 5a. Culture Positive Cases: Males } \\
\hline
\end{tabular}

\begin{tabular}{|c|c|c|c|}
\hline Ward & Klebsiella & Acinetobacter & Others \\
\hline PICU and NICU & 1 & 1 & 0 \\
\hline ICU & 1 & 2 & 0 \\
\hline \multicolumn{2}{|r|}{ Table 5b. Culture Positive Cases: Females } \\
\hline
\end{tabular}

There are some interesting findings which must be mentioned. According to Table 2, laryngeal aspirate is $100 \%$ (1 out of 1 ) culture positive, tracheal aspirate is $50 \%$ ( 1 out of 2) culture positive and ET tube tips 79\% (22 out of 28) culture positive. According to Table 3, Klebsiella pneumoniae is isolated in largest number. This data corroborates previous data as seen in a study by Dipti Chandra et al in 2017.

Next comes Acinetobacter baumannii followed by Proteus mirabilis, Citrobacter spp. and Pseudomonas aeruginosa. Remarkably, Gram positive organisms have not been isolated from these specimens in our study.

Table No. 5 shows that PICU and NICU provide a niche for Klebsiella pneumoniae and Acinetobacter baumannii. One reason for that may be because the patients in NICU and PICU are extremely vulnerable because of their age and correspondingly low immune status. The incidence ratio of Klebsiella and Acinetobacter in male and female is not significant in this study.

\section{DISCUSSION}

To conclude this study, we delved into a few well-known reference books to find out WHY certain organisms were being repeatedly isolated from endotracheal tube tips and aspirates all over India and abroad. No one particular reason was found, but the logical conclusions we could draw from our researches were as follows $8,9,10$ :

1. Klebsiella Pneumoniae: Capsule, klebocin, heat-stable toxin, lipopolysaccharide and adhesion mechanisms were all responsible for making this organism practically invincible. The adhesion mechanisms were both fimbrial i.e. mannose sensitive, haemagglutination type I, type II and KPF 28. Non-fimbrial adhesion factors were CF29K and CS31A, which are plasmid encoded. The function of enterobactin and plasmid encoded aerobactin are still being researched.

2. Acinetobacter baumannii: This organism is well known for its virulence. The important factors responsible are adhesions, invasiveness, cytotoxicity through mitochondrial damage, lipopolysaccharide and presence of siderophores. Citrobacter spp. possess adhesion mechanism with mannose sensitive type I fimbriae.

3. Proteus mirabilis: Damages epithelium by producing urea, stimulates chemotaxis, causes calcium dependent and independent haemolysis, produces IgA ase and protease.

4. Pseudomonas aeruginosa: Produces chemotactic motility, colonisation due to the presence of pili and polar flagella. This bacterium is notorious for toxin mediated immune evasion. Non-diffusible exotoxin S, U, T2Y injected via type III secretary system helps to evade phagocytosis. Diffusible exotoxin, protease, phospholipase, haemolysin, elastase and pyocyanin, through the type III protein synthesis process. For this bacterium the host inflammatory response is mediated by TLR 4 and TLR 5 . Pigments and a wide range of temperature tolerated by this organism also helps in pathogenicity. 
All the organisms mentioned above are excellent Biofilm ${ }^{11,12}$ producers. The mechanism may be different, but the net effect is the same. Acinetobacter baumannii secretes BAP or biofilm associated protein, which is regulated by BFM RS. A 2D proteomic analysis of pellicle forming Acinetobacter baumannii shows over-expression of Car-O which is an Opr-D homolog, helping biofilm formation. K1 capsular polysaccharide inhibits phagocytosis. Omp38 protein causes apoptosis of host cells. In case of Klebsiella pneumoniae, biofilm formation on abiotic surfaces is facilitated by MrkA type III fimbria and biofilm formation on human cells needs MrkD type III fimbria. TreC and SugE genes affect biofilm modulating CPS production.

Polymicrobial biofilm formation is also seen with combination of Klebsiella pneumoniae and Pseudomonas aeruginosa and Klebsiella pneumoniae and Proteus mirabilis.

Last but not the least is drug resistance ${ }^{13,14}$ seen in all these organisms individually and more pronounced in the Biofilm community. All these factors are responsible for the huge percentage of infections in the ET tubes.

\section{CONCLUSION}

Our study results show that none of the infecting organisms were Gram positive. All our isolates were Gram negative bacilli. Klebsiella pneumoniae, Proteus mirabilis, Citrobacter spp. belong to the family enterobacteriaceae and Pseudomonas aeruginosa and Acinetobacter baumannii belong to the non-fermenters. All these organisms are highly equipped to form Biofilms on abiotic surfaces as mentioned above. Their virulence factors are also quite formidable. This study aims to point out and prime the medical fraternity to remain aware of this problem and to start appropriate management as soon as infection is suspected, keeping in mind the Gram reaction and drug resistance pattern of the bacteria commonly isolated.

\section{REFERENCES}

[1] Chandra D, Laghawe A, Sadawarte $K$, et al. Microbiological profile and antimicrobial sensitivity pattern of endotracheal tube aspirates of patients in ICU of tertiary care hospital in Bhopal, India. Int J Curr Microbiol App Sci 2017;6(3):891-5.

[2] Patil T. The study of the organisms colonizing trachea in mechanically ventilated patients admitted in the Intensive Care Unit (ICU). International J Med Sci Education 2014;1(1):39-48.
[3] Mouli HCC, Nagaraja M, Chandra A, et al. Endotracheal tube tip culture in post-operative respiratory infections in open heart surgery patients: a one year prospective study. J Clin Sci Res 2016;5:214-20.

[4] Cook D, Mandell L. Endotracheal aspiration in the diagnosis of ventilator-associated pneumonia. Chest 2000;117(4 Suppl 2):195S-7S.

[5] Ananthnarayan R, Paniker CKJ. Text book of Microbiology. 10 ${ }^{\text {th }}$ edn. India: Universities Press Pvt Ltd., 2016: p. 77.

[6] Chastre J, Fagon JY. Ventilator-associated pneumonia. Am J Respir Crit Care Med 2002;165(7):867-903.

[7] Brain M. Topley and Wilson microbiology and microbial infection. $10^{\text {th }}$ edn. Hodder Arnold Publication 2010:1:B1 149-50, B1432-3.

[8] Koneman EW, Allen SD, Janda WM, et al. In: Color atlas and text book of diagnostic microbiology. $5^{\text {th }}$ edn. Philadelphia, PA: Lippincott/ The Williams and Wilkins Co., 1997.

[9] Darouiche RO. Device-associated infections: a macro problem that starts with microadeherence. Clin Infect Dis 2001;33(9):1567-72.

[10] Longo D, Fauci A, Kasper D, et al. Harrison's Principles of internal medicine. $18^{\text {th }}$ edn. McGraw-Hill Education 2011;1:756, 839, 944.

[11] Mandell G, Bennett J, Dolin R. Mandell G, Douglas and Bennett's Principles and practice of infectious

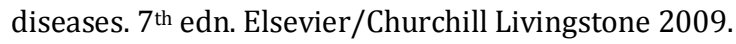

[12] Murray P, Rosenthal K, Pfaller M. Medical micro biology: with student consult. $7^{\text {th }}$ edn. Elsevier 2015.

[13] Bhattacharya P, Penmetcha U, Myneni RB, et al. Bacterial profile and antimicrobial susceptibility pattern in catheter-related nosocomial infections in a tertiary care teaching hospital, Chinakakani, Guntur district, Andra Pradesh, South India. Int J Health Sciences and Research 2015;5(6):220-35.

[14] Clinical and Laboratory Standard Institute. Performance standards for antimicrobial susceptibility testing, CLSI document M100-22, Wayne, PA: CLSI 2017. 